\title{
Routine endoscopy reduces gastric bypass complication, morbidity rates
}

Published online: 17 September 2012

(C) Springer Healthcare 2012

medwireNews: The complication/failure and morbidity rates of laparoscopic Roux-en-Y gastric bypass (LRYGB) can be substantially reduced by the routine use of intraoperative endoscopy with the linear anastomosis technique, the results of a US study indicate.

Andrew Averbach and colleagues from Saint Agnes Hospital in Baltimore, Maryland, say: "It is believed that endoscopic intraoperative evaluation of the gastrojejunal anastomosis (GJA) reduces the potential rate of anastomotic complications by more than $50 \%$."

Noting that routine endoscopic use will add an average of 5 to 10 minutes to the procedure, they continue in Obesity Surgery: "In general, routine use of intraoperative endoscopic assessment of GJA... is probably a less involved management strategy and provides a simpler approach for both the patient and treating surgeon."

Reviewing the records of 2311 patients who underwent LRYGB between 2002 and 2011, the team found that intraoperative esophagogastroenteroscopy could not be completed in three patients and was associated with iatrogenic injury in a further three patients, which was managed conservatively and not associated with delayed morbidity. The overall failure and morbidity rate was therefore $0.26 \%$.

Intraoperative air leaks were found in 80 (3.5\%) patients, which was persistent in 46 (2.0\%) patients and transient at only high insufflation pressure in 34 patients. Clinical leaks were detected in four (0.2\%) patients, two of which were initially intraoperative. Reconstruction was required due to the anastomosis being two tight in two cases.

Postoperatively, 25 (1.1\%) cases of GJA strictures were detected at 90 days. There was no correlation with patient age, gender, body mass index, or comorbidities. Total GJA-related postoperative morbidity occurred in $31(1.3 \%)$ patients, including two cases of faulty construction, four postoperative leaks, and 25 early strictures.

Without the use of intraoperative endoscopy, the team calculated that anastomosis-related morbidity would have occurred in 50 patients, which, with 25 cases of strictures, would have brought the postoperative GJA-related morbidity rate to $3.2 \%$.

By Liam Davenport, medwireNews Reporter

\section{Reference}

Obes Surg 2012; Advance online publication 